\title{
Rapid olfactory discrimination learning in adult zebrafish
}

\author{
lori Namekawa $\cdot$ Nila R. Moenig ${ }^{1,2} \cdot$ Rainer W. Friedrich ${ }^{1,2}(\mathbb{0}$
}

Received: 4 May 2018 / Accepted: 2 August 2018 / Published online: 7 August 2018

(c) The Author(s) 2018

\begin{abstract}
The zebrafish is a model organism to study olfactory information processing, but efficient behavioral procedures to analyze olfactory discrimination and memory are lacking. We devised an automated odor discrimination task for adult zebrafish based on olfactory conditioning of feeding behavior. Presentation of a conditioned odor (CS+), but not a neutral odor (CS-) was followed by food delivery at a specific location. Fish developed differential behavioral responses to CS+ and CS- within a few trials. The behavioral response to the CS+ was complex and included components reminiscent of food search such as increased swimming speed and water surface sampling. Appetitive behavior was therefore quantified by a composite score that combined measurements of multiple behavioral parameters. Robust discrimination behavior was observed in different strains, even when odors were chemically similar, and learned preferences could overcome innate odor preferences. These results confirm that zebrafish can rapidly learn to make fine odor discriminations. The procedure is efficient and provides novel opportunities to dissect the neural mechanisms underlying olfactory discrimination and memory.
\end{abstract}

Keywords Zebrafish $\cdot$ Olfaction $\cdot$ Behavior $\cdot$ Discrimination $\cdot$ Learning

\section{Introduction}

The zebrafish is an important vertebrate model to study neuronal circuit structure and function (Sumbre and de Polavieja 2014). Zebrafish larvae are small and relatively transparent, allowing for high-resolution optical measurements of neuronal activity throughout most of the brain. Even in juvenile and adult fish, activity patterns throughout major brain areas can be measured and manipulated by optical methods (Aoki et al. 2013; Fajardo et al. 2013; Jetti et al. 2014; Portugues et al. 2013; Rupprecht et al. 2016; Zhu et al. 2012). The small size of the zebrafish brain is also advantageous for dense reconstructions of neuronal connectivity (Friedrich et al. 2013; Wanner et al. 2016) and for genetic or chemical screens (MacRae and Peterson 2015). Zebrafish thus provide

Iori Namekawa and Nila R. Moenig contributed equally to this work.

Rainer W. Friedrich

Rainer.Friedrich@fmi.ch

1 Friedrich Miescher Institute for Biomedical Research, Maulbeerstrasse 66, 4058 Basel, Switzerland

2 University of Basel, 4003 Basel, Switzerland unique opportunities for quantitative analyses of brain function in wild-type animals and in genetic disease models.

To understand the neural basis of behavior, it is important to quantify behaviors of interest. At embryonic and early larval stages, zebrafish show primarily reflex-like sensorymotor behaviors with a limited potential for plasticity. Social behaviors and an increasing potential for associative learning emerge at later larval and juvenile stages (Buske and Gerlai 2011; Dreosti et al. 2015; Valente et al. 2012). Adult zebrafish and other teleosts show complex innate and cognitive behaviors (Abril-de-Abreu et al. 2015; Arganda et al. 2012; Brown et al. 2006; Buske and Gerlai 2011; Chou et al. 2016; Kalueff et al. 2013; Saverino and Gerlai 2008; Yabuki et al. 2016) including place preference and associative learning in different sensory modalities (Agetsuma et al. 2010; Aoki et al. 2013; Braubach et al. 2009; Darland and Dowling 2001; Doyle et al. 2017; Eddins et al. 2009; Lau et al. 2006; Mueller and Neuhauss 2012; Sison and Gerlai 2010; $\mathrm{Xu}$ et al. 2007). While various quantitative procedures have been established to analyze sensory-motor behaviors of zebrafish larvae, there is an increasing demand for methods to study complex behaviors and learning in juvenile or adult zebrafish.

Adult zebrafish have been used to study principles of information processing in olfaction (Friedrich 2013). 
Imaging experiments demonstrated that odors evoke scattered but non-random spatial patterns of activity across the olfactory glomeruli, the input channels of the olfactory bulb (Friedrich and Korsching 1997). Neuronal circuits within the olfactory bulb decrease the overlap between activity patterns representing similar odors and stabilize odor representations against variations in stimulus intensity (Friedrich 2013; Friedrich and Laurent 2001; Niessing and Friedrich 2010; Zhu et al. 2013). Hence, processing of odor-evoked activity patterns in the olfactory bulb may support the classification of odor representations in higher brain areas. Further studies provide insights into transformations of spatio-temporal activity patterns between the output neurons of the olfactory bulb, the mitral cells, and telencephalic area Dp, the homolog of olfactory cortex (Blumhagen et al. 2011; Jacobson et al. 2018; Yaksi et al. 2009). To explore the impact of these computations on behavior, quantitative procedures are desired to analyze odor discrimination behavior and associative learning in adult fish. Ideally, such procedures should be automated and simple to implement in a standard fish facility.

Adult zebrafish can learn to associate different sensory stimuli with reward or punishment (Agetsuma et al. 2010; Aoki et al. 2013; Braubach et al. 2009; Darland and Dowling 2001; Doyle et al. 2017; Eddins et al. 2009; Lau et al. 2006; Mueller and Neuhauss 2012; Sison and Gerlai 2010; Xu et al. 2007). Two procedures have been described for olfactory conditioning of appetitive behaviors in adult zebrafish. Based on a procedure developed for catfish (Valentinčič et al. 2000), Valentinčič and colleagues paired the infusion of an odor into a home tank with food delivery $90 \mathrm{~s}$ later (Miklavc and Valentinčič 2012). After approximately 30 training trials, the frequency of large-angle turns during the 90 s period after odor onset was significantly higher than the turn rate evoked by non-trained odors. Braubach et al. developed an appetitive olfactory conditioning procedure that follows a similar rationale (Braubach et al. 2009, 2011). The results obtained using these approaches showed that zebrafish can learn olfactory associations and discriminations. However, both approaches require substantial technical resources that are not available in a standard zebrafish facility. Moreover, most experiments did not analyze odor discrimination. We therefore sought to develop a procedure for olfactory discrimination learning that is simple to implement and automate.

To minimize stress, we chose an appetitive rather than an aversive conditioning paradigm. Odor discrimination tasks for rodents often involve unfamiliar behavioral components such as nose pokes or lever presses (Bodyak and Slotnick 1999; Frederick et al. 2009; Kay and Laurent 1999; Rinberg et al. 2006). We avoided such unfamiliar components and conditioned a familiar feeding behavior on an olfactory cue in an environment similar to the home tank. One odor stimulus (CS+) was followed by a food reward while another stimulus (CS-) had no consequence. A closely related strategy has recently been successful in auditory and visual conditioning of adult zebrafish without discrimination training (Doyle et al. 2017). Our behavioral paradigm includes the analysis of multiple behavioral components and resulted in rapid and robust olfactory discrimination learning, thus providing a basis for mechanistic analyses of olfactory processing and associative learning in zebrafish.

\section{Methods}

\section{Animals}

Zebrafish (Danio rerio) were raised and kept as groups in a standard facility at $26.5-27.5^{\circ} \mathrm{C}$ on a $14 / 10 \mathrm{~h}$ light/dark cycle. Fish were 7-10 months old and not selected for sex. Different wild-type strains and transgenic lines were used in separate cohorts. Experiments 1 and 3 were performed using wild-type fish (Abek $\times$ WIK). Experiments 2 and 4 were performed using fish that expressed halorhodopsin fused to YFP [eNpHR3.0YFP; (Gradinaru et al. 2010)] in GABAergic neurons. Experiment 5 was performed using a mixture of different wild-type and transgenic fish. All experimental protocols were approved by the Veterinary Department of the Kanton Basel-Stadt (Switzerland).

\section{Experimental setup and odor application}

Throughout the experiment, fish were kept individually in tanks that were usually custom-made from flat transparent polystyrole to avoid optical distortions by curved surfaces (typical dimensions: $29 \times 9.5 \times 7 \mathrm{~cm}$; height of the water column: ca. $6 \mathrm{~cm}$ ). The tank was divided by a mesh into a front compartment containing the fish $(\sim 10 \times 20 \mathrm{~cm})$ and a rear compartment containing the suction tube for water removal $(\sim 9 \times 10 \mathrm{~cm})$. A feeding ring with a diameter of $\sim 4.5 \mathrm{~cm}$ was made of silicon tubing and floated in a front corner (Fig. 1a). Food was delivered into the feeding ring from a remote location through a feeding tube. In most experiments, food was pushed down the tube into the feeding ring by a custom device that applied computer-controlled pulses of pressurized air to the food delivery tube. In a subset of experiments, food was manually blown into the feeding ring using a plastic Pasteur pipette. Visual contact between the fish and the experimenter was avoided. Food was either a mixture of Gemma Micro 300 (Skretting) and crushed food flakes (Tetramin, Tetra) or powder food (SDS100). Food was delivered in small portions that were usually consumed within $<30 \mathrm{~s}$.

Tanks were perfused with water from the fish facility using a peristaltic pump at a flow rate of $25 \mathrm{ml} / \mathrm{min}$. The flow 

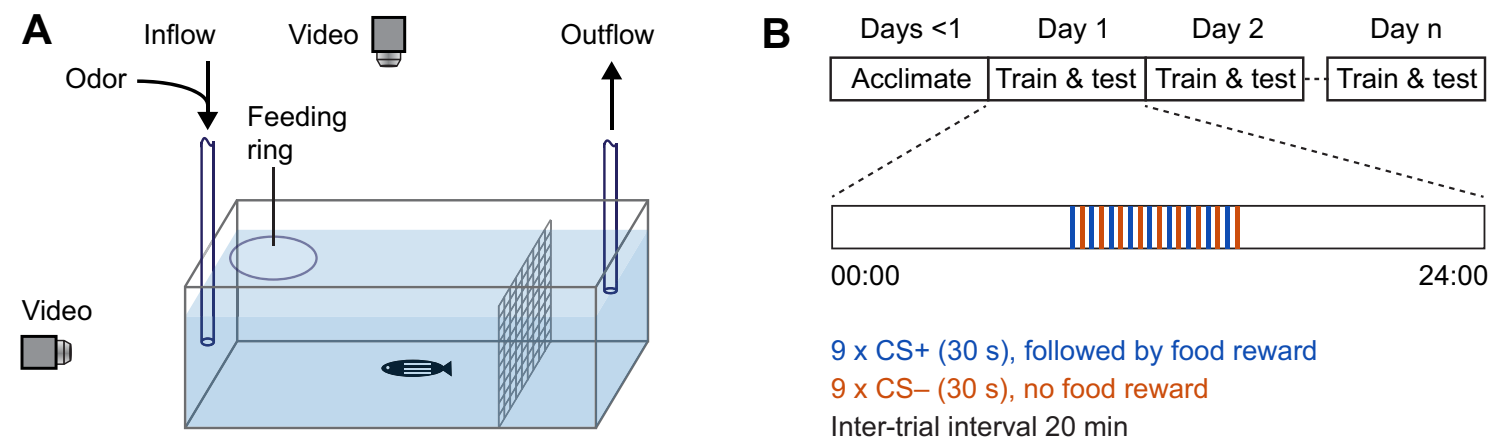

Fig. 1 Experimental setup and protocol. a Schematic of experimental setup. b Experimental schedule

was constantly on for approximately $7 \mathrm{~h}$ on each day during the period when odor and food stimuli were delivered. Outside this time window and during the acclimation period the flow was off. Water entered the tank through an inflow tube (inner diameter, $1.6 \mathrm{~mm}$ ) in the front approximately at half-height in the water column. Water was removed from the tank by suction through an outflow tube at the rear of the tank and discarded. To minimize fluctuations of the water level, a pipette tip $(200 \mu \mathrm{l})$ was attached to the tip of suction tube and positioned at the desired height.

Olfactory stimuli were amino acids (Ala, Trp or Cys; Sigma Aldrich, $\geq 98 \%$ purity), which are natural odorants for aquatic animals (Carr 1988). Odors were prepared freshly on each day by diluting a stock solution $\left(6 \times 10^{-3} \mathrm{M}\right)$ in fish water to a concentration of $6 \times 10^{-5} \mathrm{M}$. Odors were delivered into the tank by switching the perfusion for $30 \mathrm{~s}$ from a reservoir-containing fish water to a reservoir-containing odor solution. Switching was achieved using computer-controlled, motorized valves (WTA-2K-3MFE-3, Takasago Electric, Inc.) and valve controllers (ValveLink8.2® Controller, AutoMate Scientific). Video analyses using a dye showed that the stimulus distribution during the first $30 \mathrm{~s}$ of application was inhomogeneous and discontinuous with highest concentrations near the inflow tube. Subsequently, the stimulus distribution equilibrated throughout the tank. The nominal odor concentration assuming even dilution in the tank was $\sim 4.5 \times 10^{-8} \mathrm{M}$, which is at or below the detection threshold of adult zebrafish for amino acids (Michel and Lubomudrov 1995; Miklavc and Valentinčič 2012). Hence, stimulus concentration was reduced to levels below threshold by dilution and water exchange (Miklavc and Valentinčič 2012).

Experiments were performed in up to four tanks in parallel, each containing a single fish. Tanks were separated by opaque screens, illuminated from below by infrared light, and filmed simultaneously by two orthogonal cameras (Fig. 1a). Sufficient video quality was achieved using standard web cameras at video rate (30 frames per second). Simple devices such as tennis balls or shock absorbers were used to partially isolate the tabletop from vibrations. The setup was placed inside an opaque enclosure which was lined with sound-absorbing foam in most experiments.

\section{Experimental schedule}

Fish remained in the experimental tanks after transfer from the facility for the duration of the experiment. During an initial acclimatization period of 1-3 days, fish received neither food nor odor stimuli. When training started, odors were applied at inter-trial intervals (ITIs) of $20 \mathrm{~min}$. No specific cue was given to signal trial onset. One odor (conditioned stimulus, CS+) was followed by food application (unconditioned stimulus, US) $30 \mathrm{~s}$ after stimulus onset while a second odor (CS-) was not followed by food application. Usually, a total of nine CS+ and nine CS- were delivered per day in alternating fashion (Fig. 1b). Hence, training extended over a period of $6 \mathrm{~h} /$ day, usually starting at approximately 09:00 h. Fish were trained for a total of 2-9 days. During training, behavior was quantified in each trial during the $30 \mathrm{~s}$ period between odor onset and food delivery. Four fish of the same strain and age were usually trained in parallel on the same two odors A and B. Training was balanced such that two fish received odor $\mathrm{A}$ as $\mathrm{CS}+$ and $\mathrm{B}$ as $\mathrm{CS}-$ while assignments were reversed for the other two fish.

\section{Quantitative analysis of behavior}

Orthogonal video recordings were analyzed to track the 3D position of each fish as a function of time (Fig. 2a). In each frame, the fish was segmented and represented by a point at the center of gravity. The following behavioral parameters were then extracted from the 3D trajectories: (1) Swimming speed. Instantaneous swimming speed was calculated as the displacement of fish between successive video frames. (2) Relative z-position. This parameter quantifies the relative position in the water column along the z-direction. 0 represents the bottom of the tank; 1 represents the water surface. (3) Residence in the reward zone. This binary parameter is 1 when the fish is located 

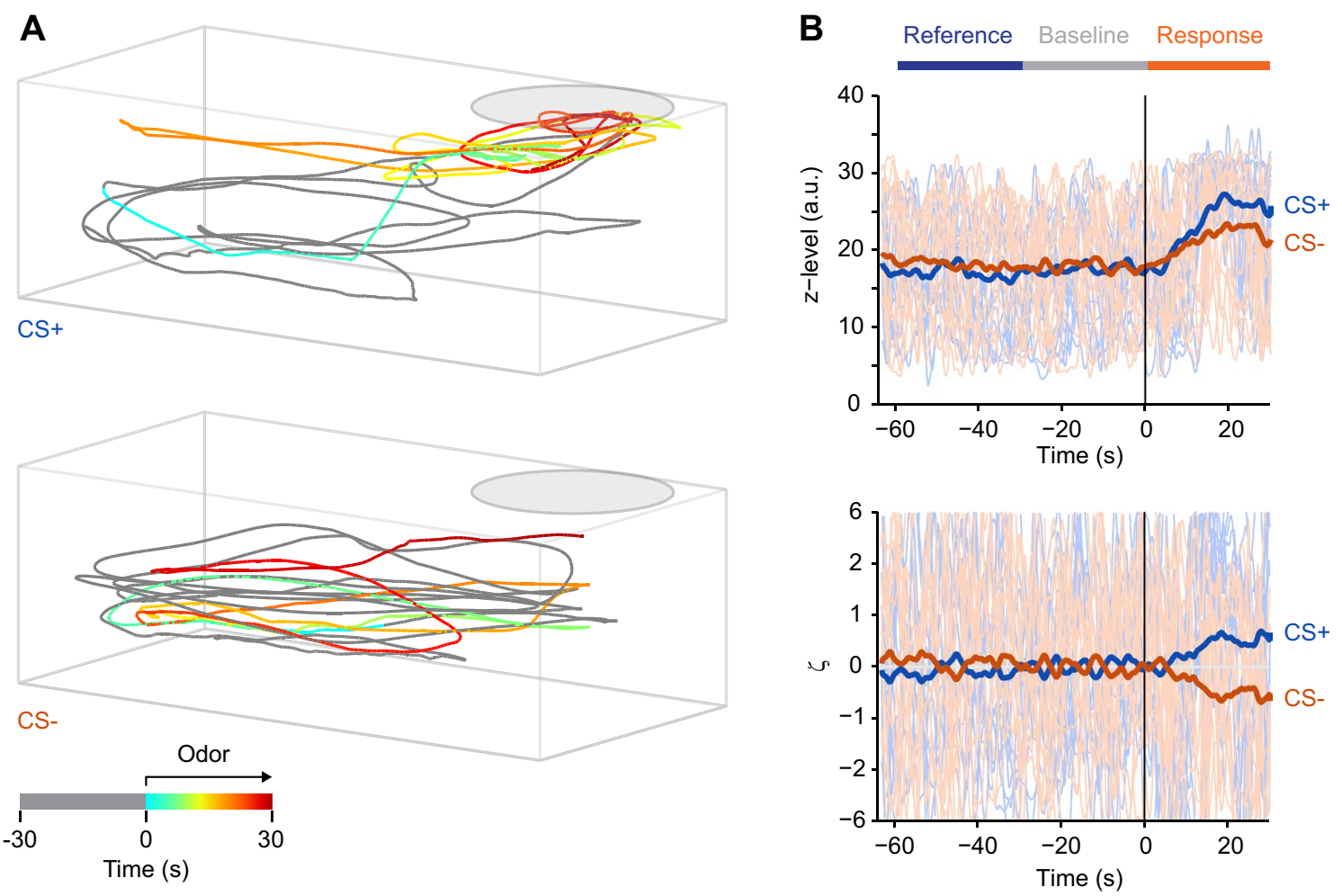

Fig. 2 Analysis of behavior. a Examples of swimming trajectories prior to and during presentation of CS+ (top; Trp) and CS- (bottom; Ala) on day 5 of training. Each trajectory is $60 \mathrm{~s}$ long, comprising $30 \mathrm{~s}$ before and $30 \mathrm{~s}$ after odor onset, prior to food delivery. Time is color coded; pre-odor time is gray. b Top: time series showing one

in the reward zone and 0 otherwise. The reward zone is defined as a rectangular area bounding the feeding ring and spanning the shorter axis of the experiment tank, covering approximately one-third of the tank's footprint. (4) Surface sampling events. A surface sampling event is defined as the crossing of a threshold in the $\mathrm{z}$ dimension below the center of the feeding ring. The threshold was set at approximately $70 \%$ of the height of the water column. Surface sampling events are usually discrete and occur when fish collect food from the water surface but are rare otherwise. (5) Distance to inflow. This is the 3D distance between the fish and the opening of the inflow tube. (6) Circling. This behavior refers to stereotyped circular swimming along the walls of the tank. We observed that such a swimming pattern is sometimes maintained for extended periods of time when fish are undisturbed but the behavior is interrupted by salient sensory input. To quantify circling, we computed power spectra from measurements of the position along the long axis of the tank using a $30 \mathrm{~s}$ Tukey window and quantified the relative power in a low-frequency regime that corresponded to the frequency of circular swimming $(0.029-0.146 \mathrm{~Hz})$ in 1-s time bins. Python-based software for automated video analysis is behavioral variable (z-level) as a function of time for all trials in an experiment; thick lines show averages. Odor was delivered at $t=0$. Bottom: Same time series after transformation to $\zeta$ scores. Horizontal bars indicate reference, baseline and response time windows

available at https://github.com/i-namekawa/TopSideMon itor. Because the analysis of behavior was fully automated, it was not performed blindly.

Measurements of each behavioral parameter in each video frame yielded one time series of values per parameter and trial. We defined three time windows for analysis: (1) Baseline time window: This window comprised the $30 \mathrm{~s}$ prior to odor onset and served as a baseline in each trial. (2) Response time window: This window was defined as the time between odor onset and the time of food delivery in $\mathrm{CS}+$ trials or the equivalent time in CS- trials. This time window was usually $30 \mathrm{~s}$ long and used to assess behavioral responses to odor stimulation. (3) Reference time window. This time window was defined as a time window immediately prior to the baseline window with a length equal to the response window. This window served to assess trial-to-trial variability of behavior in the absence of stimulation. Note that the response window and the reference window were equidistant in time from the baseline window.

Because spontaneous behavior can fluctuate between trials, we quantified the change in parameter values relative to a baseline in each trial. The baseline was defined as the mean value of a parameter during the baseline time window 
and subtracted from the time series. Time series were then averaged over all CS+ and over all CS- trials, and the mean of these averages was subtracted from each individual time series. To quantify trial-to-trial variability in the absence of stimulation, the standard deviation of the time-averaged values during the reference window across trials was calculated. To normalize mean-subtracted time series to this variability measure in each fish, all time series from a given fish were divided by this standard deviation. As a consequence, the family of time series for each behavioral parameter was centered on zero and normalized by a measure of pre-stimulus variability (Fig. 2b). We refer to these transformed time series values as $\zeta$ scores because they are closely related to Z-scores: positive values represent behavioral responses larger than the mean response to all stimuli, negative values represent behavioral responses smaller than the mean, and the absolute value reflects response magnitude relative to a variability measure. $\zeta$ scores differ from $z$-scores because they are normalized to the variability during a fixed prestimulus time window. As a consequence, $\zeta$ scores reflect the change in behavior relative to a baseline but they cannot be interpreted quantitatively as a $\mathrm{z}$-score.

To obtain a single measure per trial, $\zeta$ scores were timeaveraged over the response time window. The sequence of these averaged $\zeta$ scores over successive trials is referred to as a trial series of $\zeta$ scores.

\section{Results}

\section{Olfactory discrimination learning: method and basic observations}

We developed an experimental paradigm to establish associations between odors and food. Individual zebrafish were trained in tanks with continuous water perfusion and a feeding ring floating in one corner (Fig. 1a). Fish were acclimated to the training tanks for at least $24 \mathrm{~h}$ prior to training and kept in the training tank throughout the experiment. Every $20 \mathrm{~min}$, one of two amino acid odors (CS+, $\left.\mathrm{CS}-; 6 \times 10^{-5} \mathrm{M}\right)$ was introduced into the perfusion for $30 \mathrm{~s} \mathrm{(Fig.} \mathrm{1b)} \mathrm{and} \mathrm{slowly} \mathrm{removed} \mathrm{thereafter} \mathrm{by} \mathrm{dilution} \mathrm{and}$ water exchange (Methods). Thirty seconds after the onset of the $\mathrm{CS}+$, a few small food pellets were delivered into the feeding ring while the $\mathrm{CS}-$ was not rewarded. Usually, nine trials with each stimulus were alternated on each day. We hypothesized that fish learn to respond to the $\mathrm{CS}+$, but not to the $\mathrm{CS}-$, with anticipatory food search behavior.

Fish were tracked in 3D using two orthogonal video cameras and custom software. In trained fish, swimming trajectories showed obvious differences after delivery of the $\mathrm{CS}+$ or CS- (Fig. 2a). Upon presentation of the CS+, fish often increased their swimming speed and spent more time near the feeding ring, consistent with previous observations in other olfactory conditioning paradigms (Braubach et al. 2009; Miklavc and Valentinčič 2012). In addition, fish often elevated their position in the water column, sampled the water surface as during feeding, and sometimes approached the inflow tube. Stereotyped cyclic swimming along the wall of the experiment tank, which usually occurred during interstimulus intervals, was often interrupted. These behavioral changes were less pronounced or absent upon presentation of the CS-

To quantify these observations, we analyzed parameters of the 3D swimming trajectory that reflect different behavioral components. Analysis tools were developed to automatically quantify (1) the instantaneous swimming speed ("Speed"), (2) the relative height of the fish in the water column ("z-level"), (3) the distance of the fish to the water inflow ("Distance"), (4) the frequency of surface sampling events ("Surface"), (5) the probability that the fish is located in a rectangular area around the feeding ring ("Area"), and (6) the amount of stereotyped, cyclic swimming ("Circling"). For each parameter and trial, time series of measurements were obtained. To transform measurements into values related to $z$-scores, we defined three time windows, each usually $30 \mathrm{~s}$ long: a "baseline time window" immediately before stimulus onset, a "response time window" immediately following the baseline time window, and a "reference time window" immediately preceding the baseline time window (Fig. 2b). Measured values were transformed by (1) subtraction of the mean value during the baseline time window, (2) subtraction of the time series averaged over all trials, and (3) normalization to the inter-trial variability in the reference time window (Methods). This transformation results in a dimensionless measure that is normalized to prestimulus variability and centered on the mean at each time point (Fig. 2b). Because this measure is closely related to a $\mathrm{z}$-score, we refer to it as $\zeta$ score (Methods). The signs of $\zeta$ scores for "Distance" and "Circling" were inverted so that positive (negative) $\zeta$ values always reflected responses that were more (less) appetitive than the mean for all parameters. To analyze learning curves over trials, $\zeta$ scores were time-averaged during the $30 \mathrm{~s}$ between odor onset and food delivery ("response time window") to obtain one value per trial. Medians were then calculated over the trials of each day to obtain one value per day.

\section{Experiment 1: extended discrimination training}

We initially trained 12 fish using Ala and Trp as odor stimuli (experiment 1; $9 \mathrm{CS}+$ trials and $9 \mathrm{CS}$ - trials per day). Fish were trained for a total of nine days (81 trials for each stimulus). Assignments of odors as CS+ or CS- were balanced over fish. $\zeta$ scores of all parameters were significantly 

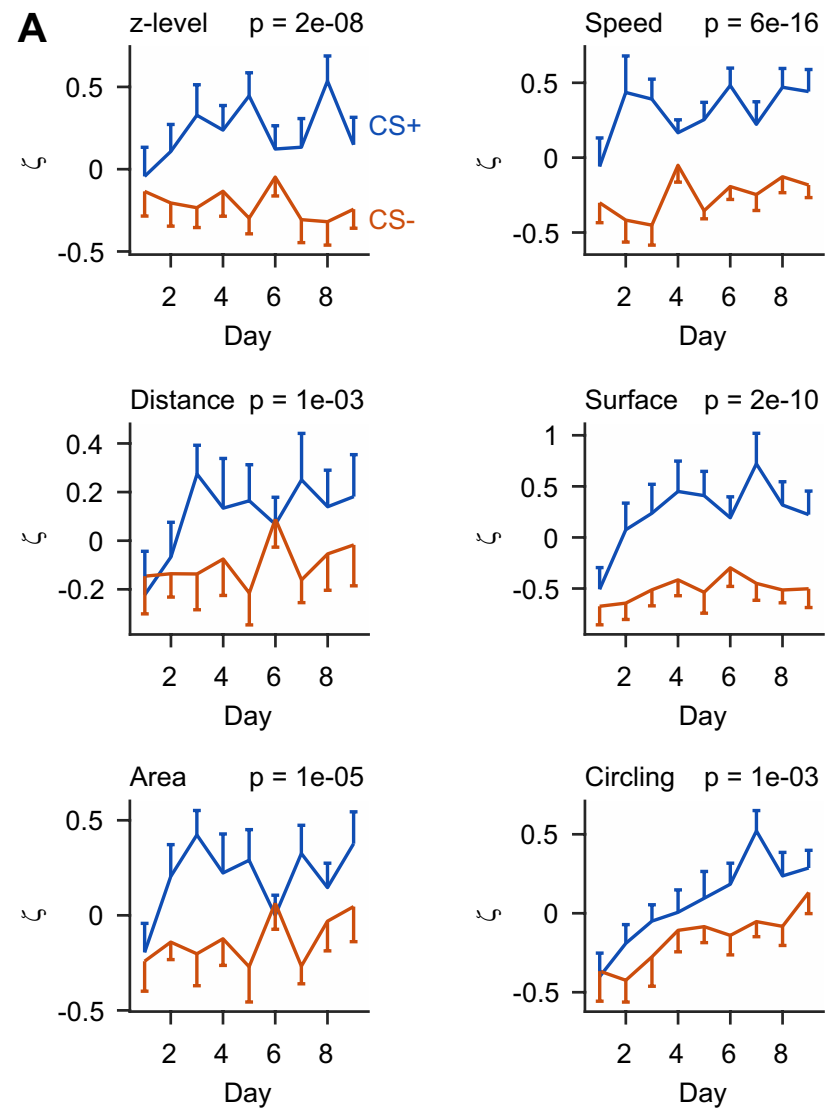

B
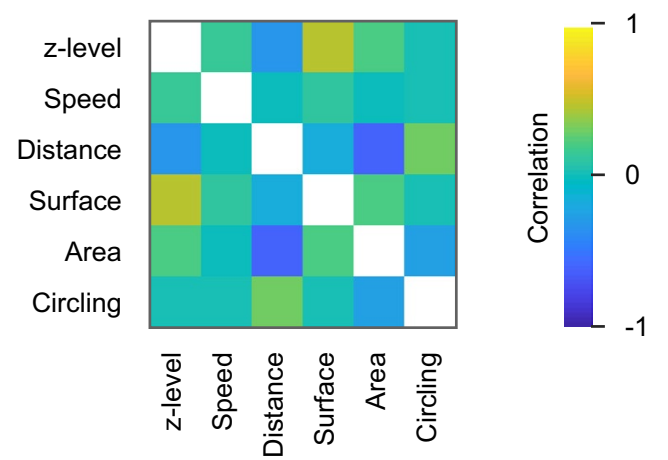

Fig. 3 Analysis of discrimination learning. a $\zeta$ scores for the six behavioral parameters as a function of time, binned per day (medians) and averaged over fish ( $n=12$ fish). $P$ values show that responses to $\mathrm{CS}+$ and CS- were significantly different (Wilcoxon rank-sum test comparing all individual trials). Assignment of odor stimuli (Ala and $\mathrm{Trp}$ ) as CS+ and CS- was varied between individuals. b Mean correlation between time series of $\zeta$ scores for different behavioral parameters ( $n=62$ fish from experiments $1-3$ ). Correlations were calculated on a trial-by-trial basis for each fish and averaged over fish

different between $\mathrm{CS}+$ and CS - trials (Fig. 3a). Hence, fish learned to discriminate between the $\mathrm{CS}+$ and the $\mathrm{CS}-$.

Visual observations of behaving fish further suggested that the contribution of different components to the overall behavioral response varied substantially between trials and individuals. For example, a fish may respond to the $\mathrm{CS}+$ primarily with fast swimming in one trial but with surface sampling in the next trial. Consistent with this observation, trial-by-trial correlations between most parameters were relatively low (Fig. 3b; mean correlation coefficient \pm SD: $0.00 \pm 0.27$ ). Hence, combining multiple behavioral measurements may improve the detection of learned behavior because different parameters convey non-redundant information. Alternatively, combining measurements may increase noise. To explore these possibilities, we computed a composite measure of behavior, $\zeta_{\text {comp }}$, based on Stouffer's method for the combination of z-scores (Stouffer et al. 1949). As in Stouffer's method, $\zeta_{\text {comp }}$ is the sum over all $\zeta$ scores normalized by the square root of the number of $\zeta$ scores $(n=6)$. This procedure enhanced, rather than decreased, the statistical significance (Fig. 4a). Hence, the combined analysis of multiple behavioral parameters enhanced the detection of learning-induced changes in behavior, presumably because the conditioned behavior comprises multiple components.

The detection of learned behaviors may be further optimized by taking the covariances between behavioral parameters into account. To test this hypothesis, we weighted each parameter by the ratio of its auto-covariance to the sum of its auto- and cross-covariances (Stouffer et al. 1949). Covariances were determined using all fish in experiments 1-3 $(n=62)$. The resulting combined score $\zeta_{\text {comp,weighted }}$ is thus corrected for redundant information in individual parameters. This approach further enhanced the separation of behavioral scores for the CS+ and CS- (Fig. 4b). However, the enhancement was minor, consistent with the modest cross-correlation between behavioral response components (Fig. 3b). The non-weighted $\zeta_{\text {comp }}$ therefore offers the opportunity to obtain a highly informative combined behavioral score without the need to determine a behavioral covariance matrix, which is labor-intensive. As this approach is likely to be chosen in future applications, further analyses used the non-weighted measure $\zeta_{\text {comp }}$.

Odor discrimination at the end of training was quantified by the difference $d$ between $\zeta_{\text {comp }}$ for the CS+ and CS-, averaged over the last 12 trials. After 9 days of training, $\mathrm{d}$ was significantly different from zero $(d=0.98 \pm 0.28$; mean $\pm \mathrm{SEM} ; n=12$ fish; $P=0.005$; Wilcoxon signed rank test), confirming that fish discriminated between the CS+ and CS-.

Behavioral responses to the CS + and CS - deviated already during the first day of training and approached saturation after approximately 3 days (Fig. 4a). Differences between behavioral scores for the $\mathrm{CS}+$ and the $\mathrm{CS}-$ were statistically significant already on day 2 , and the discrimination score $d$ was statistically significant already when all data after day 2 were omitted $(d=0.66 \pm 0.26$; mean \pm SEM; $n=12$ fish; $P=0.03$; Wilcoxon signed rank test). Hence, 
Fig. 4 Discrimination learning curve. a Mean composite behavioral scores $\zeta_{\text {comp }}$ for responses to the CS+ and CS- as a function of training days (experiment 1). Data points for each day show the median of nine trials, averaged over $n=12$ fish. Error bars show SEM over fish. $P$ value: Wilcoxon rank-sum test comparing all individual $\mathrm{CS}+$ and $\mathrm{CS}-$ trials, pooled over all days. Circles depict significant differences between behavioral responses to the $\mathrm{CS}+$ and $\mathrm{CS}-$ on each day (Wilcoxon rank-sum test comparing all individual trials on a given day). Open circles: $0.01 \leq P<0.05$; filled circles: $P<0.01$. Assignment of odor stimuli (Ala and Trp) as CS+ and CS- was varied between individuals. b Same as above for the combined behavioral score

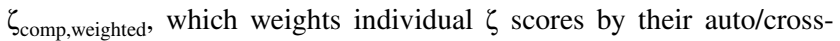
covariances. c Quantification of behavioral responses to the CS+ and $\mathrm{CS}-$ in successive trials during the first 2 days of training, averaged over all fish in experiments $1-3$ ( $n=62$ fish; mean \pm SEM). The first two trials and the last two trials were omitted because individual trial series of $\zeta$ scores were median filtered with window size five to minimize the potential impact of outliers. Open and filled circles depict significant differences between behavioral responses to the CS+ and CS - as in a. Assignment of odor stimuli (Ala and Trp) as CS+ and $\mathrm{CS}-$ was varied between individuals

fish learned to discriminate between odors after few training trials.

\section{Experiments 2 and 3: shorter discrimination training}

To corroborate these results, we trained 36 additional fish on the discrimination of Ala and Trp for 27-36 trials (experiment 2). Again, fish showed significantly different behavioral responses to the CS+ and CS- at the end of training, with a discrimination score similar to that observed in experiment $1(d=1.14 \pm 0.17$; mean $\pm \mathrm{SEM} ; n=36$ fish; $P=3 \mathrm{e}-7$, Wilcoxon signed rank test; Fig. 5).

In addition, another 14 fish were trained on the discrimination of Ala and Trp for a total of 18 trials each (experiment 3) and showed significant discrimination behavior at the end of training $(d=0.51 \pm 0.15$; mean $\pm \mathrm{SEM} ; n=14$ fish; $P=0.002$, Wilcoxon signed rank test). These results were pooled with the first $18 \mathrm{CS}+$ and $18 \mathrm{CS}-$ trials from experiments 1 and 2 to obtain a large sample $(n=62$ fish). The mean discrimination score in this sample was $(d=0.76 \pm 0.12$; mean \pm SEM; $p=2 \mathrm{e}-7$, Wilcoxon signed rank test; Fig. 5). The large sample size was exploited to analyze the initial phase of the learning curve at single-trial resolution. To minimize the impact of outliers, trial series of $\zeta_{\text {comp }}$ scores were median-filtered using a window size of five trials. The mean behavioral scores $\zeta_{\text {comp }}$ of responses to the CS+ and CS - gradually diverged and became statistically different at trial six (median-filtered data from trials 4-8; Fig. 4c). Hence, significant discrimination behavior emerged already during the first day of training.

Behavioral scores in experiments 1-3 were similar although fish were from different strains and included transgenics (see "Methods"). Hence, behavioral results are unlikely to be sensitive to the genetic background. No
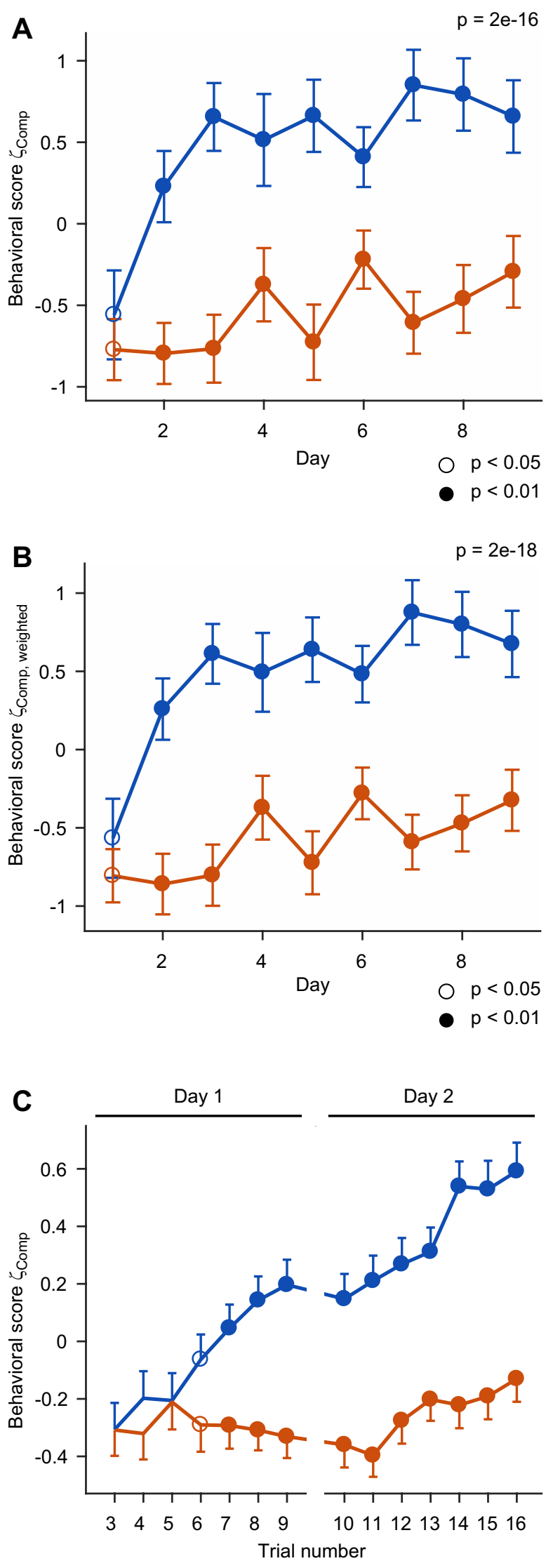


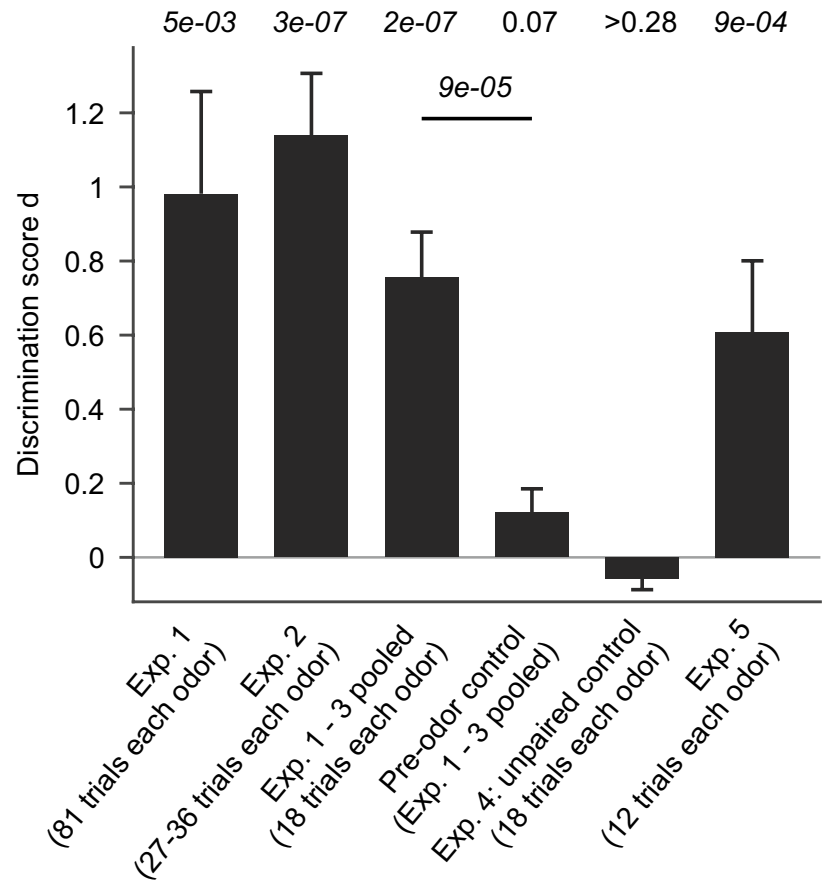

Fig. 5 Quantification of discrimination behavior. Bars show the discrimination score $d$ (mean \pm SEM) obtained in different experiments. $P$ values are from a Wilcoxon signed rank test comparing the observed discrimination score $\mathrm{d}$ against the null hypothesis that $d=0$. Horizontal line shows statistical comparison between the discrimination score d obtained in pooled experiments $1-3$ (first 18 trials only) and the $\mathrm{d}$ value of the corresponding pre-odor control (Wilcoxon rank-sum test)

significant differences were observed between males and females in any experimental group.

\section{Experiment 4: unpaired control}

Differential responses to the $\mathrm{CS}+$ and $\mathrm{CS}-$ in experiments 1 and 2 cannot be explained by innate odor preferences because amino acid stimuli were balanced across fish, implying that the observed behavior reflects associative learning. To confirm this conclusion, we performed two controls. First, we analyzed behavior in the absence of a conditioned stimulus by re-analyzing swimming trajectories from experiments 1-3 using the time window 60-30 s before odor application as the response time window. The baseline and reference time windows were shifted accordingly. The resulting value of $\mathrm{d}$ was not significantly different from zero $(d=0.12 \pm 0.07$; mean $\pm \mathrm{SEM} ; n=62$ fish; $P=0.07$, Wilcoxon signed rank test) and significantly different from the discrimination score of stimulus-induced behavior in the same fish $(P=9 \mathrm{e}-5$; Fig. 5). Hence, the observed discrimination behavior cannot be explained by a bias in the training or analysis procedure.
Second, we exposed an additional 16 fish to 18 presentations of Ala and 18 presentations of Trp as before ( 9 trials each per day; 2 days total). However, food was delivered 15 min after application of both odors with a probability of $50 \%$. Hence, fish were exposed to the same number of odor stimuli and food deliveries as before but odors did not predict food. We then randomly assigned Ala or Trp as CS+ and CS- in different fish in a balanced fashion and analyzed behavioral responses as before. The procedure was repeated 10 times for different random stimulus assignments. No significant discrimination score was obtained in any of these assignments $(P>0.28$ in all cases; mean \pm SD: $P=0.64 \pm 0.22$; range $0.28-0.87)$ and the discrimination score remained close to zero $(d=-0.06 \pm 0.10$; mean \pm SD; Fig. 5). These results support the conclusion that the differential behavioral responses in experiments $1-3$ reflect associative learning.

\section{Experiment 5: conditioning can overcome innate preferences}

We further examined whether the learned association between an odor stimulus and a food reward can overcome innate odor responses. A previous study reported that naïve zebrafish are attracted to Ala but repelled by Cys (Vitebsky et al. 2005). We tested whether the aversive response to Cys can be overcome when zebrafish are trained in the odor discrimination paradigm with Cys as CS+ and Ala as CS- (6 fish) or the opposite assignment (8 fish). Each fish received only 12 trials with each stimulus, distributed over 3-5 days. Nevertheless, training resulted in the emergence of positive behavioral responses to Cys and odor discrimination was statistically significant ( $d=0.61 ; n=14$ fish; $P=9 \mathrm{e}-4$; Fig. 5). Hence, associative appetitive conditioning can also be performed using aversive odors.

\section{Discussion}

We developed efficient methods to analyze olfactory discrimination learning in adult zebrafish. Fish learned to respond selectively to one of two odor stimuli with anticipatory appetitive behavior. Training and analysis procedures are automated and can be performed in a standard laboratory environment. This paradigm is well-suited to analyze odor discrimination behavior and the underlying neuronal mechanisms in zebrafish.

\section{Odor discrimination paradigm}

Olfactory conditioning resulted in complex modifications of behavioral responses to odors. Learned responses to the CS+ included an increase in swimming velocity, an 
approach of the reward zone, and sampling of the water surface, reminiscent of the behavior that fish display in their home tanks when caretakers approach for feeding. Hence, fish appeared to learn the association between an olfactory cue and a familiar set of feeding-related behaviors. We therefore assume that our training procedure resulted primarily in classical conditioning.

Previous procedures for olfactory conditioning of adult zebrafish are difficult to implement in a standard laboratory setting because they require enormous amounts of water (Braubach et al. 2009) or very large tanks (Miklavc and Valentinčič 2012). Moreover, training and analysis were time-consuming because procedures are not automated. Importantly, previous procedures did not involve differential conditioning to two odors (CS+ and CS-), with few exceptions (Miklavc and Valentinčič 2012). We therefore developed a procedure that is fully automated, requires only standard resources, and includes discrimination training. We expect that this procedure will be valuable to analyze the neural basis of discrimination learning.

A recent study by Doyle and colleagues described automated procedures for auditory and visual conditioning of adult zebrafish that also rely on the conditioning of feeding behavior (Doyle et al. 2017). The tasks follow a similar rationale as ours but exhibit differences in experimental procedures. First, we did not maintain fish in the fish facility during training, mainly to facilitate odor application and to avoid contamination of the circulating water with odors. Second, we trained fish individually rather than in groups. Training one fish per tank decreases throughput but facilitates quantitative analyses of multiple behavioral components and enables comparisons between individuals. Third, we trained fish to discriminate between two sensory cues (CS+ and CS-), rather than to associate a single cue with a behavioral output, because differential conditioning is desired to analyze the neural basis of sensory discrimination. Despite the differences in task design and sensory modalities, fish rapidly learned associations between the CS and US in both studies (Doyle et al. 2017). An appetitive paradigm for operant visual discrimination learning, in contrast, required more extensive training (Mueller and Neuhauss 2012), possibly because fish needed to learn a novel behavioral sequence to collect rewards. Hence, classical conditioning of feeding behavior appears to be an effective strategy for the design of appetitive learning tasks in zebrafish.

Based on observations of behaving fish, we hypothesized that the conditioned behavior consists of multiple behavioral components, and that the contribution of different behavioral components varies between trials and individuals. Consistent with this observation, the combined analysis of multiple behavioral components improved the detection of learned behavioral responses. Hence, the overt behavior was complex even though the conditioning paradigm is conceptually simple.

\section{Olfactory discrimination learning}

Zebrafish developed differential behavioral responses to related amino acid odors when and only when they differed in their prediction of food. Hence, fish discriminated between odors and learned specific associations between odors and behaviors, consistent with previous findings (Braubach et al. 2009; Miklavc and Valentinčič 2012). Appetitive olfactory conditioning could be achieved even using an odor that is innately aversive. Hence, olfactory conditioning is a robust phenomenon that can overcome innate odor preferences. This approach may be exploited to dissect neural pathways that mediate innate and learned behavioral responses to odors.

The 30 s delay between the onset of the CS and US provided the opportunity to quantify behavioral responses in each trial. Continuous learning curves could therefore be acquired without the need to introduce separate probe trials, which may result in extinction. As the acquisition of memory can be sensitive to the precise temporal relationship between the CS and the US, experimental conditions may be further improved by optimizing the delay between odor and food application. However, we did not attempt this because the chosen protocol already resulted in robust conditioning.

Statistically different behavioral responses to the CS+ and CS- were detected already after few training trials. Zebrafish can therefore establish specific olfactory memories based on a small amount of experience. Odor discrimination tasks that are widely used in rodents, in contrast, often require hundreds of training trials to reach asymptotic performance (Abraham et al. 2004; Bodyak and Slotnick 1999; Rinberg et al. 2006). The reason for this difference in training requirement remains unclear. One possibility is that the tasks for rodents include unnatural behavioral components such as nose pokes, whereas the task for fish is based on the conditioning of familiar food search behavior.

The ability of animals to discriminate odors is usually assessed by a behavioral readout that is based on odor discrimination learning. However, because learning itself modifies odor representations (Abraham et al. 2014; Chapuis and Wilson 2011; Chu et al. 2016; Li et al. 2008; Yamada et al. 2017), mechanisms of odor discrimination in naïve or nearly naïve animals remain difficult to analyze. A rapid conditioning procedure may open new opportunities to address this issue. We anticipate that the combination of behavioral and physiological analyses in zebrafish will provide new insights into the neuronal basis of olfactory processing and memory.

Acknowledgements We thank G. Jacobson for helpful input and support and the Friedrich lab for stimulating discussions. This work 
was supported by the Novartis Research Foundation, by the Deutsche Forschungsgemeinschaft (DFG; FR 1667/2-2), by the Swiss National Science Foundation (SNF; 31003A_135196 and 310030B_1528331), and by the European Research Council (ERC) under the European Union's Horizon 2020 research and innovation programme (Grant Agreement No 742576).

Open Access This article is distributed under the terms of the Creative Commons Attribution 4.0 International License (http://creativeco mmons.org/licenses/by/4.0/), which permits unrestricted use, distribution, and reproduction in any medium, provided you give appropriate credit to the original author(s) and the source, provide a link to the Creative Commons license, and indicate if changes were made.

\section{References}

Abraham NM, Spors H, Carleton A, Margrie TW, Kuner T, Schaefer AT (2004) Maintaining accuracy at the expense of speed: stimulus similarity defines odor discrimination time in mice. Neuron 44:865-876

Abraham NM, Vincis R, Lagier S, Rodriguez I, Carleton A (2014) Long term functional plasticity of sensory inputs mediated by olfactory learning. eLife 3:e02109 https://doi.org/10.7554/eLife .02109

Abril-de-Abreu R, Cruz AS, Oliveira RF (2015) Social dominance modulates eavesdropping in zebrafish. R Soc Open Sci 2:150220. https://doi.org/10.1098/rsos.150220

Agetsuma $\mathrm{M}$ et al (2010) The habenula is crucial for experiencedependent modification of fear responses in zebrafish. Nat Neurosci 13:1354-1356. https://doi.org/10.1038/nn.2654

Aoki $\mathrm{T}$ et al (2013) Imaging of neural ensemble for the retrieval of a learned behavioral program. Neuron 78:881-894. https://doi. org/10.1016/j.neuron.2013.04.009

Arganda S, Perez-Escudero A, de Polavieja GG (2012) A common rule for decision making in animal collectives across species. Proc Natl Acad Sci USA 109:20508-20513. https://doi.org/10.1073/ pnas. 1210664109

Blumhagen F, Zhu P, Shum J, Zhang Schärer Y-P, Yaksi E, Deisseroth K, Friedrich RW (2011) Neuronal filtering of multiplexed odour representations. Nature 479:493-498. https://doi.org/10.1038/ nature 10633

Bodyak N, Slotnick B (1999) Performance of mice in an automated olfactometer: odor detection, discrimination and odor memory. Chem Senses 24:637-645

Braubach OR, Wood HD, Gadbois S, Fine A, Croll RP (2009) Olfactory conditioning in the zebrafish (Danio rerio). Behav Brain Res 198:190-198

Braubach OR, Wyeth RC, Murray A, Fine A, Croll RP (2011) A simple and effective method to condition olfactory behaviors in groups of zebrafish. In: Kalueff AV, Cachat JM (eds) Zebrafish neurobehavioral protocols. Humana Press, New York City, pp 85-98

Brown C, Laland K, Krause J (eds) (2006) Fish cognition and behavior. Blackwell Publishing, Oxford

Buske C, Gerlai R (2011) Shoaling develops with age in Zebrafish (Danio rerio). Prog Neuro-psychopharmacol Biol Psychiatry 35:1409-1415. https://doi.org/10.1016/j.pnpbp.2010.09.003

Carr WES (1988) The molecular nature of chemical stimuli in the aquatic environment. In: Atema J, Fay RR, Popper AN, Tavolga WN (eds) Sensory biology of aquatic animals. Springer, New York, pp 3-27
Chapuis J, Wilson DA (2011) Bidirectional plasticity of cortical pattern recognition and behavioral sensory acuity. Nat Neurosci 15:155161. https://doi.org/10.1038/nn.2966

Chou MY et al (2016) Social conflict resolution regulated by two dorsal habenular subregions in zebrafish. Science 352:87-90. https://doi. org/10.1126/science.aac9508

Chu MW, Li WL, Komiyama T (2016) Balancing the robustness and efficiency of odor representations during learning. Neuron 92:174-186. https://doi.org/10.1016/j.neuron.2016.09.004

Darland T, Dowling JE (2001) Behavioral screening for cocaine sensitivity in mutagenized zebrafish. Proc Natl Acad Sci USA 98:11691-11696

Doyle JM et al (2017) A simple automated system for appetitive conditioning of zebrafish in their home tanks. Behav Brain Res 317:444-452. https://doi.org/10.1016/j.bbr.2016.09.044

Dreosti E, Lopes G, Kampff AR, Wilson SW (2015) Development of social behavior in young zebrafish. Front Neural Circuits 9:39. https://doi.org/10.3389/fncir.2015.00039

Eddins D, Petro A, Williams P, Cerutti DT, Levin ED (2009) Nicotine effects on learning in zebrafish: the role of dopaminergic systems. Psychopharmacology 202:103-109. https://doi.org/10.1007/s0021 3-008-1287-4

Fajardo O, Zhu P, Friedrich RW (2013) Control of a specific motor program by a small brain area in zebrafish. Front Neural Circuits 7:67

Frederick DE, Barlas L, Ievins A, Kay LM (2009) A critical test of the overlap hypothesis for odor mixture perception. Behav Neurosci 123:430-437

Friedrich RW (2013) Information processing in the olfactory system of zebrafish. Annu Rev Neurosci 36:383-402

Friedrich RW, Korsching SI (1997) Combinatorial and chemotopic odorant coding in the zebrafish olfactory bulb visualized by optical imaging. Neuron 18:737-752

Friedrich RW, Laurent G (2001) Dynamic optimization of odor representations in the olfactory bulb by slow temporal patterning of mitral cell activity. Science 291:889-894

Friedrich RW, Genoud C, Wanner AA (2013) Analyzing the structure and function of neuronal circuits in zebrafish. Front Neural Circuits $7: 71$

Gradinaru V et al (2010) Molecular and cellular approaches for diversifying and extending optogenetics. Cell 141:154-165

Jacobson GA, Rupprecht P, Friedrich RW (2018) Experience-dependent plasticity of odor representations in the telencephalon of Zebrafish. Curr Biol 28:1-14 e13. https://doi.org/10.1016/j. cub.2017.11.007

Jetti SK, Vendrell-Llopis N, Yaksi E (2014) Spontaneous activity governs olfactory representations in spatially organized habenular microcircuits. Curr Biol 24:434-439. https://doi.org/10.1016/j. cub.2014.01.015

Kalueff AV et al (2013) Towards a comprehensive catalog of zebrafish behavior 1.0 and beyond Zebrafish 10:70-86 https://doi. org/10.1089/zeb.2012.0861

Kay LM, Laurent G (1999) Odor- and context-dependent modulation of mitral cell activity in behaving rats. Nat Neurosci 2:1003-1009

Lau B, Bretaud S, Huang Y, Lin E, Guo S (2006) Dissociation of food and opiate preference by a genetic mutation in zebrafish. Genes Brain Behav 5:497-505. https://doi.org/10.1111/j.1601183X.2005.00185.x

Li W, Howard JD, Parrish TB, Gottfried JA (2008) Aversive learning enhances perceptual and cortical discrimination of indiscriminable odor cues. Science 319:1842-1845

MacRae CA, Peterson RT (2015) Zebrafish as tools for drug discovery. Nat Rev Drug Discov 14:721-731. https://doi.org/10.1038/ $\operatorname{nrd} 4627$

Michel WC, Lubomudrov LM (1995) Specificity and sensitivity of the olfactory organ of the zebrafish, Danio rerio. J Comp Physiol A 177:191-199 
Miklavc P, Valentinčič T (2012) Chemotopy of amino acids on the olfactory bulb predicts olfactory discrimination capabilities of zebrafish Danio rerio. Chem Senses 37:65-75. https://doi. org/10.1093/chemse/bjr066

Mueller KP, Neuhauss SCF (2012) Automated visual choice discrimination learning in zebrafish (Danio rerio). J Integr Neurosci 11:73-85. https://doi.org/10.1142/S0219635212500057

Niessing J, Friedrich RW (2010) Olfactory pattern classification by discrete neuronal network states. Nature 465:47-52

Portugues R, Severi KE, Wyart C, Ahrens MB (2013) Optogenetics in a transparent animal: circuit function in the larval zebrafish. Curr Opin Neurobiol 23:119-126. https://doi.org/10.1016/j. conb.2012.11.001

Rinberg D, Koulakov A, Gelperin A (2006) Speed-accuracy tradeoff in olfaction. Neuron 51:351-358

Rupprecht P, Prendergast A, Wyart C, Friedrich RW (2016) Remote z-scanning with a macroscopic voice coil motor for fast 3D multiphoton laser scanning microscopy. Biomed Opt Express 7:1656-1671

Saverino C, Gerlai R (2008) The social zebrafish: behavioral responses to conspecific, heterospecific, and computer animated fish. Behav Brain Res 191:77-87. https://doi.org/10.1016/j.bbr.2008.03.013

Sison M, Gerlai R (2010) Associative learning in zebrafish (Danio rerio) in the plus maze. Behav Brain Res 207:99-104. https://doi. org/10.1016/j.bbr.2009.09.043

Stouffer SA, Suchman EA, DeVinney LC, Star SA, Williams RMJ (1949) The American soldier, vol.1: adjustment during army life. Princeton University Press, Princeton

Sumbre G, de Polavieja GG (2014) The world according to zebrafish: how neural circuits generate behavior. Front Neural Circuits 8:91. https://doi.org/10.3389/fncir.2014.00091

Valente A, Huang KH, Portugues R, Engert F (2012) Ontogeny of classical and operant learning behaviors in zebrafish. Learn Mem 19:170-177. https://doi.org/10.1101//m.025668.112
Valentinčič T, Metelko J, Ota D, Pirc V, Blejec A (2000) Olfactory discrimination of amino acids in brown bullhead catfish. Chem Senses 25:21-29

Vitebsky A, Reyes R, Sanderson MJ, Michel WC, Whitlock KE (2005) Isolation and characterization of the laure olfactory behavioral mutant in the zebrafish, Danio rerio. Dev Dyn 234:229-242

Wanner AA, Genoud C, Masudi T, Siksou L, Friedrich RW (2016) Dense EM-based reconstruction of the interglomerular projectome in the zebrafish olfactory bulb. Nat Neurosci 19:816-825. https:// doi.org/10.1038/nn.4290

Xu X, Scott-Scheiern T, Kempker L, Simons K (2007) Active avoidance conditioning in zebrafish (Danio rerio). Neurobiol Learn Mem 87:72-77

Yabuki Y et al (2016) Olfactory receptor for prostaglandin F2alpha mediates male fish courtship behavior. Nat Neurosci 19:897-904. https://doi.org/10.1038/nn.4314

Yaksi E, von Saint Paul F, Niessing J, Bundschuh ST, Friedrich RW (2009) Transformation of odor representations in target areas of the olfactory bulb. Nat Neurosci 12:474-482

Yamada Y, Bhaukaurally K, Madarasz TJ, Pouget A, Rodriguez I, Carleton A (2017) Context- and output layer-dependent long-term ensemble plasticity in a sensory circuit. Neuron 93:1198-1212 e1195. https://doi.org/10.1016/j.neuron.2017.02.006

Zhu P, Fajardo O, Shum J, Zhang Schärer Y-P, Friedrich RW (2012) High-resolution optical control of spatiotemporal neuronal activity patterns in zebrafish using a digital micromirror device. Nat Protoc 7:1410-1425. https://doi.org/10.1038/nprot.2012.072

Zhu P, Frank T, Friedrich RW (2013) Equalization of odor representations by a network of electrically coupled inhibitory interneurons. Nat Neurosci 16:1678-1686. https://doi.org/10.1038/nn.3528 